\section{ARRHYTHMIAS \\ CPAP WITH ABLATION REDUCES AF IN OSA}

Continuous positive airway pressure (CPAP) ventilation reduces the risk of arrhythmia recurrence after catheter ablation pulmonary vein isolation (PVI) among patients with atrial fibrillation (AF) and obstructive sleep apnoea (OSA). In addition, PVI has minimal benefit in these patients, unless they are compliant with CPAP therapy. These findings from Fein et al. have been published in JACC.

The association between OSA and AF is well known, and OSA is thought to modify the atrial substrate structurally and electrically through various mechanisms. Fein et al. conducted a retrospective analysis of patients with OSA and AF who underwent PVI to determine whether CPAP therapy reduced the rate of AF recurrence after ablation.

Data from 32 patients who received daily CPAP for 3 months before PVI and throughout follow-up, and 30 patients who did not undergo CPAP, were analyzed. At 1 year after $\mathrm{PVI}$, survival free from $\mathrm{AF}$ was $71.9 \%$ among 'CPAP users' and $36.7 \%$ in 'non-users' $(P=0.01)$. Notably, among 30 control patients without OSA who also underwent $\mathrm{PVI}$ for $\mathrm{AF}$, survival without $\mathrm{AF}$ recurrence was similar to that of the CPAP users. Among the patients with OSA who underwent PVI, arrhythmia-free survival without antiarrhythmic drugs or repeat ablation was higher among CPAP users than non-users $(65.6 \%$ vs $33.3 \%, P=0.02)$. Compared with individuals without OSA who underwent PVI, the CPAP users had a similar risk of recurrent arrhythmia after $\mathrm{PVI}$, whereas the individuals who did not receive CPAP were more than twice as likely to experience recurrent AF. Indeed, the patients with OSA who did not use CPAP had a similar rate of $A F$ recurrence after $P V I$ as 22 CPAP-using patients with AF who did not undergo PVI but were managed medically. Fein et al. conclude that "unless a patient is 'optimized' from the standpoint of OSA, there may be little value in pursuing invasive treatment procedures."

The investigators call for a randomized trial of CPAP therapy in patients with OSA and AF. This appeal is echoed by Dr Bernard Belhassen in an accompanying editorial. He writes that the study has important clinical implications and that "confirmation of these results in randomized controlled trials will certainly represent a great advance in our management of patients with AF and OSA".

Alexandra King

Original article Fein, A. S. et al. Treatment of obstructuve sleep apnea reduces risk of atrial fibrillation recurrence following catheter ablation. J. Am. Coll. Cardiol. doi:10.1016/j.jacc.2013.03.052 